\title{
Nutritional Science
}

National Cancer Institute

\section{Source}

National Cancer Institute. Nutritional Science. NCI Thesaurus. Code C16927.

The science of food, the nutrients and other substances contained therein, their action, interaction, and balance in relation to health and disease. 